\title{
miR-181b as a key regulator of the oncogenic process and its clinical implications in cancer (Review)
}

\author{
JUAN LIU ${ }^{1}$, WEIFENG SHI ${ }^{1}$, CHANGPING WU ${ }^{1}$, JINGFANG JU ${ }^{2}$ and JINGTING JIANG ${ }^{1}$ \\ ${ }^{1}$ Department of Tumor Biological Treatment, The Third Affiliated Hospital of Soochow University, Changzhou, \\ Jiangsu 213003, P.R. China; ${ }^{2}$ Department of Pathology, Stony Brook University, Stony Brook, NY 11794, USA
}

Received September 03, 2013; Accepted October 04, 2013

DOI: $10.3892 /$ br.2013.199

\begin{abstract}
MicroRNAs (miRNAs or miRs) are small, non-coding, single-stranded RNA molecules that regulate gene expression at the post-transcriptional level to repress protein expression of target genes. Among these, miR-181b has been found to be a critical regulatory miRNA linking inflammation and cancer. The functional significance of miR-181b in various tumors and translational research suggests that it exhibits great potential as a predictive and prognostic biomarker. Extensive efforts are underway to identify mRNA targets and the affected regulatory networks, which may be the key to providing a better understanding of miR-181b-mediated signaling pathways.
\end{abstract}

\section{Contents}

1. Introduction

2. miR-181b provides a critical link between inflammation and cancer

3. Functional significance and clinical relevance of miR-181b in cancer

4. Conclusion

\section{Introduction}

MicroRNAs (miRNAs or miRs) have been identified as an important class of endogenously expressed small RNAs with critical regulatory functions that modulate protein synthesis rate (1-3). The functional miRNA that targets mature mRNA is single-stranded and typically 19-22-nt long after being processed from double-stranded RNA. miRNA

Correspondence to: Professor Jingting Jiang, Department of Tumor Biological Treatment, The Third Affiliated Hospital of Soochow University, 185 Juqian Road, Changzhou, Jiangsu 213003, P.R. China

E-mail: jjtnew@163.com

Key words: miRNA, miR-181b, oncogene, tumor suppressor gene, regulator molecules regulate gene expression mainly by binding to complementary sequences in the 3'-untranslated region of target mRNAs and the formation of RNA-induced silencing complexes, suppressing mRNA translation or degrading the miRNA-bound mRNA transcripts (4). The functional significance of miRNAs was initially demonstrated in the developmental process of Caenorhabditis elegans $(5,6)$. Additional studies confirmed a strong association between miRNA and cancer (7-11). Cancer is characterized by uncontrolled cell growth, high proliferation, anti-apoptosis, acquisition of invasive properties to adjacent tissues and organs and inappropriate cell survival (12-14). Epigenetic change is crucial in tumorigenesis and miRNAs are key components of epigenetic alterations (15). With the expansion of miRNA expression profiling efforts, miRNA expression patterns have been identified as unique signatures associated with diagnosis, staging, progression, prognosis and response to treatment. In addition, miRNAs are directly involved in cancer development by activating oncogenic pathways or suppressing tumor suppressor gene pathways or protein expression (16-19). Among these, miR-181b was identified as one of the most important miRNAs contributing to tumor initiation and progression.

\section{2. miR-181b provides a critical link between inflammation and cancer}

miR-181 family members contain four highly conserved mature miRNAs: miR-181a, -181b, -181c and -181d (20). These are independently derived from six precursors located on three different chromosomes: miR-181a-1 and -181b-1 are located on chromosome 1; miR-181a-2 and -181b-2 are located on chromosome 9; miR-181c and -181d are located on chromosome 19; miR-181b-1 and -181b-2 are identical in their mature sequences, but located on different chromosomes (21). Evidence indicates that miR-181s are aberrantly expressed in tumor tissues, suggesting a potentially important role in tumor development and/or progression $(22,23)$.

miR-181b was previously shown to be highly expressed in pancreatic $(22,24)$, head and neck (25) and bladder cancer (26). By contrast, it was found to be downregulated in gastric (27) and prostate cancer (28). Those results suggested that the function of miR-181b may be unique, depending on the type of tumor and cellular context (29-31). It was previously demonstrated that miR-181b is a critical link between inflammation 
Table I. Expression of miR-181b in different types of cancer and relevant mechanism.

\begin{tabular}{|c|c|c|c|}
\hline Type of cancer & Change of miR-181b & Functions and mechanisms & Refs. \\
\hline Gastric & Downregulation & $\begin{array}{l}\text { miR-181b plays a role in the development of MDR in } \\
\text { gastric cancer cell lines by targeting BCL2 } \\
\text { miR-181b modulates CREB1 expression in human } \\
\text { gastric adenocarcinoma }\end{array}$ & $23,40,41$ \\
\hline Lung & Downregulation & $\begin{array}{l}\text { miR-181b targeting BCL2 plays a role in the development } \\
\text { of MDR in lung cancer cell lines by modulation of apoptosis }\end{array}$ & 41 \\
\hline Colon & Upregulation & p53 mutations or deletions trigger oncogene activation & $16,38,39$ \\
\hline Pancreatic & Upregulation & Unclear & 22,24 \\
\hline Head and neck & Upregulation & Unclear & 25 \\
\hline Prostate & Downregulation & Unclear & 28 \\
\hline $\mathrm{HCC}$ & Upregulation & $\begin{array}{l}\text { TIMP3, a tumor suppressor, is targeted by miR-181b } \\
\text { miR-181b enhances MMP } 2 \text { and } 9 \text { activity and promotes } \\
\text { growth, clonogenic survival, migration and invasion } \\
\text { of HCC cells }\end{array}$ & $21,36,42$ \\
\hline Breast & Upregulation & $\begin{array}{l}\text { HMGA } 1, \mathrm{CBX} 7 \text { and miR-181b may play } \\
\text { critical roles in cancer progression }\end{array}$ & 44,45 \\
\hline Thyroid & Upregulation & $\begin{array}{l}\text { miR-181b may promote cell proliferation } \\
\text { c-Kit, a target of miR-181b, may also be involved }\end{array}$ & 17 \\
\hline Oral carcinoma & Upregulation & $\begin{array}{l}\text { miR-181b may play an important role in } \\
\text { malignant transformation }\end{array}$ & 32 \\
\hline Osteosarcoma & Upregulation & Unclear & 46 \\
\hline Retinoblastoma & Upregulation & miR-181b may promote proliferation of retinoblastoma cells & 47 \\
\hline Glioma & Downregulation & $\begin{array}{l}\text { miR-181b acts as a tumor suppressor that triggers growth } \\
\text { inhibition, apoptosis and invasion inhibition in glioma cells }\end{array}$ & $48-52$ \\
\hline AML & Upregulation & $\begin{array}{l}\text { miR-181b targets MLK2, contributing to cell proliferation, } \\
\text { which plays a critical role in the progression of AML }\end{array}$ & 55 \\
\hline CLL & Downregulation & $\begin{array}{l}\text { miR-181b inhibits BCL2, MCL1 and X-linked inhibitor } \\
\text { of apoptosis protein } \\
\text { miRNAs, including miR-181b, upregulate TCL1 associated } \\
\text { with BMSCs, protecting CLL cells from apoptosis }\end{array}$ & $8,56-58$ \\
\hline
\end{tabular}

HCC, hepatocellular carcinoma; MDR, multidrug resistance; BCL2, B-cell lymphoma 2; CREB1, cAMP responsive element binding protein 1; TIMP, tissue inhibitor of metalloproteinases; HMGA1, high-mobility group AT-hook 1; CBX7, chromobox homolog 7; AML, acute myeloid leukemia; MLK2, mixed lineage kinase 2; MCL1, myeloid cell leukemia sequence 1; TCL1, T-cell leukemia/lymphoma 1; CLL, chronic lymphocytic leukemia; BMSC, bone marrow stromal cells.

and malignant transformation (32). STAT3, a transcription factor activated by IL-6 (33-35), directly activates miR-21 and -181b-1 (36). Of note, transient expression of miR-181b induces the epigenetic switch and inhibits cylindromatosis (CYLD) tumor suppressor, a target of miR-181b, from negatively modulating NF- $\kappa$ B activity. Therefore, miR-181b and CYLD are part of the positive feedback loop that underlies the epigenetic switch between inflammation and cancer $(36,37)$. The expression status of miR-181b in different types of cancer and underlying mechanisms are listed in Table I. Our current understanding of the role of miR-181b in tumorigenesis is illustrated in Fig. 1.

\section{Functional significance and clinical relevance of miR-181b in cancer}

miR-181b in colorectal cancer. One of the first clinical translational studies of miR-181b was on colorectal cancer. Compared to normal colon tissue, four highly expressed miRNAs, including miR-181b, were identified in colon cancer specimens (38). The expression level of miR-181b was significantly correlated with chemoresponse to S-1 in colorectal cancer (39). S-1 is an oral fluorouracil derivative, which comprises ftorafur, gimeracil and potassium oxonate in a molar ratio of 1:0.4:1. A number of studies reported that it 


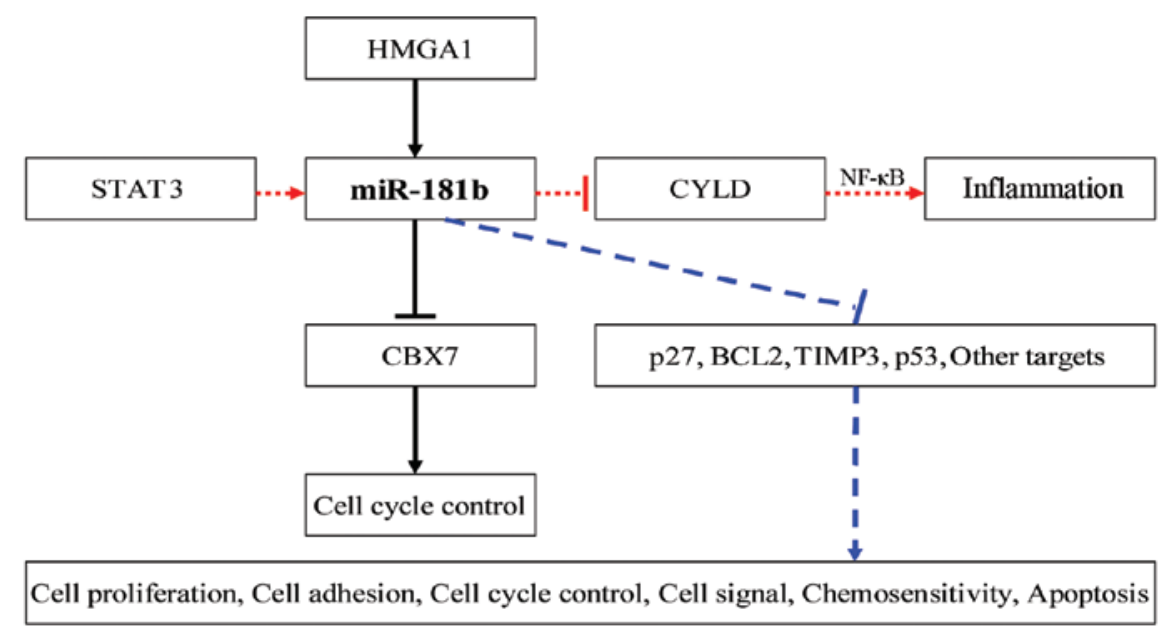

Figure 1. Schematic illustration of miR-181b-mediated targets and pathways. The expression of miR-181b is regulated by STAT3 and HMGA1. miR-181b is a critical component of inflammation and tumorigenesis by suppressing cylindromatosis (CYLD) expression. miR-181b exerts its effect on cell cycle control by suppressing chromobox homolog 7 (CBX7) and p27. miR-181b also affects cell proliferation, cell adhesion, chemosensitivity and apoptosis by regulating BCL2, TIMP3, p53 and other important targets and pathways. HMGA1, high-mobility group AT-hook 1; BCL2, B-cell lymphoma 2; TIMP, tissue inhibitor of metalloproteinases.

is safe and effective to use S-1 in the treatment of advanced colorectal cancer and late-stage gastric cancer patients $(40,41)$. It was predicted that miR-181b is able to modulate the expression of a number of genes at the translational level, such as cytochrome $c$, ECIP-1, MAPPKKK1, TEM6, E2F5, GATA6, PP2B and eIF5A. These genes may be active in cell signaling, cell cycle control and chemosensitivity in the majority of cells harbouring p53 mutations or deletions (38). In a recent study, miR-181b was shown to be inversely correlated with colon cancer patient survival (16).

miR-181b in gastric and lung cancer. The clinical significance of miR-181b was also investigated in gastric cancer patients undergoing S-1 treatment. Our recent studies demonstrated that miR-181b is overexpressed in gastric cancer compared to normal tissues (42). The efficacy of S-1 in the treatment of patients with advanced gastric cancer and patient survival were inversely correlated with the expression of miR-181b (42). Thus, miR-181b exhibits great potential as a prognostic biomarker in late-stage gastric cancer.

Another study demonstrated that miR-181b was downregulated in the multidrug-resistant (MDR) SGC7901/vincristine human gastric cancer and A549/cisplatin lung cancer cell lines and the downregulation of miR-181b was concurrent with the elevated level of BCL2 protein in these two cell lines. Those findings indicated that miR-181b may play a role in the development of MDR through targeting BCL2 in gastric and lung cancer cell lines (43).

miR-181b in hepatocellular carcinoma (HCC). miR-181b is transcriptionally activated by the $\mathrm{Wnt} / \beta$-catenin signaling pathway in HCC and the $\beta$-catenin binding site is in the promoter region of the miR-181a-2 and -181b-2 transcripts. Thus, miR-181b is highly expressed as a result of the activation of Wnt/ $/$-catenin signaling (21). Another study reported that miRNAs were deregulated during the early stages of hepatocarcinogenesis induced by a choline- and amino acid-defined diet in C57BL/6 mice. miR-181b was significantly upregulated in the livers of mice that persisted at preneoplastic stage. TIMP3, a tumor suppressor and a target of miR-181b, was significantly suppressed in the liver. By contrast, miR-181b enhanced the activity of MMP2 and 9 and promoted growth, clonogenic survival, migration and invasion of HCC cells. It was also demonstrated that miR-181b was involved in the TGFß signaling pathway (43). The overexpression of miR-181b enhanced the resistance of HCC cells to doxorubicin. Increased expression of miR-181b was detecetd in patients with non-alcoholic steatohepatitis and HCC (43). It was also recently demonstrated that miR-181b is overexpressed in the serum of patients with liver cirrhosis, targeting p27-regulated cell cycle (45). Those results suggested that miR-181b may play an important role in hepatocarcinogenesis.

$m i R-181 b$ in breast cancer. The efforts to elucidate the role of miRNAs in breast cancer were initiated with expression profiling. In order to investigate the association between clinicopathological characteristics or patient survival and the expression profile of miRNAs in primary breast cancer, the global expression profiling of miRNAs in breast cancer was investigated by a microarray expression profiling approach. The results revealed that nine miRNAs were upregulated $>2$-fold in breast cancer compared to normal adjacent tumor tissues, including miR-181b (46).

High-mobility group AT-hook 1 (HMGA1) modulates miR-181b expression by direct transcriptional activation. HMGA1 is frequently activated in several malignant tumors, including breast carcinomas (29). miR-181b is downregulated during cancer progression and targeted chromobox homolog (CBX7) negatively regulates the cell cycle at the G1 phase. In addition, HMGA1 negatively regulates CBX7 gene expression. Those results suggested that there is a novel breast cancer progression pathway, involving HMGA1, CBX7 and miR-181b (47).

miR-181b in papillary thyroid carcinoma (PTC). Expression analysis identified a subset of miRNAs that is overexpressed in 
human PTC. miR-181b, -221 and -222 in particular, were found to be highly expressed in the majority of patients with PTC, suggesting that the increasing level of miR-181b may represent a unique signature of human PTC, which may promote cell proliferation. c-Kit, which is one of the critical targets of miR-181b, may also be involved in this process (17).

miR-181b in oral carcinoma, osteosarcoma and retinoblastoma. In oral carcinoma, miR-181b, -21 and -345 were found to be consistently upregulated and were associated with the increase in lesion severity during progression. Therefore, these miRNAs may be valuable biomarkers for evaluating the risk of malignant transformation (32). miR-181b was found to be overexpressed in osteosarcoma and may exhibit potential as a critical pre-treatment biomarker of metastasis (48). Hypoxia is the basic characteristic of retinoblastoma and facilitates poor prognosis and resistance to traditional therapy. miR-181b was among the highly expressed miRNAs under hypoxic conditions and inhibition of miR-181b may suppress proliferation of retinoblastoma cells (49). Thus, miR-181b may represent a novel target for the future clinical treatment of retinoblastoma.

miR-181b in glioma. Several miRNAs are involved in the modulation of glioma development (50). miR-181b was found to be downregulated in glioma tissue (50-52). miR-181b acts as a tumor suppressor that causes growth inhibition, apoptosis and invasion inhibition in glioma cells (52). Those findings suggested that aberrantly downregulated miR-181b may be a critical factor for the appearance in human malignant glioma.

Moreover, Kaplan-Meier survival analysis demonstrated that low expression of miR-181b and $-106 \mathrm{a}$ or overexpression of miR-21 was significantly associated with poor patient survival and Cox proportional hazard regression analysis suggested that its prognostic impact is independent of other clinicopathological factors (53). A recent study demonstrated that the most significant change was the downregulation of miR-181b in patients in response to concomitant chemoradiotherapy with temozolomide (TMZ) (54). Olea europaea leaf extract (OLE) was shown to exert an anti-proliferative effect on the T98G cell line. miRNA target genes are involved in cell cycle and apoptotic pathways. miR-181b, in particular, was markedly overexpressed following treatment with TMZ and OLE (55). Thus, miR-181b may be a potential biomarker for glioma and chemotherapy treatment outcome.

miR-181b in acute myeloid leukemia (AML) and chronic lymphocytic leukemia (CLL). miRNAs have been shown to modulate disease progression in leukemia (56). miR-181b was found to be highly expressed in AML and, by targeting the mixed lineage kinase 2 (MLK2), caused cell proliferation, which is crucial in the progression of AML and provides a useful treatment biomarker (57). However, miR-181b was downregulated in CLL, which exhibits potential as a biomarker of CLL and is positively correlated with patient survival. miR-181b was also shown to inhibit BCL2 and MCL1. In cells containing wild-type p53, increasing the expression level of miR-181b markedly accelerated apoptosis $(8,58,59)$. miRNAs upregulate TCL1 associated with bone marrow stromal cells (BMSC), protecting CLL cells from apoptosis (60). Therefore, miR-181b may provide a novel target in the treatment of CLL.

\section{Conclusion}

miRNAs have a broad range of regulatory functions in the modulation of protein synthesis. Abnormal expression of miRNAs has been shown to contribute to cancer development and/or progression. Growing evidence suggests that miR-181b is one of the main factors involved in tumorigenesis, affecting several tumor types. As a result, it is crucial to further investigate the functions and underlying molecular mechanisms of miR-181b in malignant tumor transformation. By fully understanding the target genes and regulatory networks of miR-181b using the systems biology approach, we may be able to determine the potential of miR-181b as biomarker and novel therapeutic target.

\section{Acknowledgements}

This study was supported by the National Natural Science Foundation of China (nos. 81171653 and 30972703), the Natural Science Foundation of Jiangsu province (nos. BK2011246 and BK2011247), the Project of Six Batch of Major Talent Summit (no. BRA2010037) and Society Development Plans, Department of Science and Technology, Changzhou (nos. CJ20112020, CZ20110024 and CS20102020).

\section{References}

1. Lee RC, Feinbaum RL and Ambros V: The C.elegans heterochronic gene lin-4 encodes small RNAs with antisense complementarity to lin-14. Cell 75: 843-854, 1993.

2. Lagos-Quintana M, Rauhut R, Lendeckel W, et al: Identification of novel genes coding for small expressed RNAs. Science 294: 853-858, 2001.

3. Lee RC and Ambros V: An extensive class of small RNAs in Caenorhabditis elegans. Science 294: 862-864, 2001.

4. Salaun B, Yamamoto T, Badran B, et al: Differentiation associated regulation of microRNA expression in vivo in human CD8 ${ }^{+} \mathrm{T}$ cell subsets. J Transl Med 9: 44, 2011.

5. Ooi AG, Sahoo D, Adorno M, et al: MicroRNA-125b expands hematopoietic stem cells and enriches for the lymphoid-balanced and lymphoid-biased subsets. Proc Natl Acad Sci USA 107: 21505-21510, 2010.

6. Ghisi M, Corradin A, Basso K, et al: Modulation of microRNA expression in human T-cell development: targeting of NOTCH3 by miR-150. Blood 117: 7053-7062, 2011.

7. Visone R, Veronese A, Balatti V, et al: MiR-181b: new perspective to evaluate disease progression in chronic lymphocytic leukemia. Oncotarget 3: 195-202, 2012.

8. Zhu DX, Miao KR, Fang C, et al: Aberrant microRNA expression in Chinese patients with chronic lymphocytic leukemia. Leuk Res 35: 730-734, 2011.

9. Tavano F, di Mola FF, Piepoli A, et al: Changes in miR-143 and miR-21 expression and clinicopathological correlations in pancreatic cancers. Pancreas 41: 1280-1284, 2012.

10. Esquela-Kerscher A and Slack FJ: Oncomirs - microRNAs with a role in cancer. Nat Rev Cancer 6: 259-269, 2006.

11. Michael MZ, O' Connor SM, van Holst Pellekaan NG, et al: Reduced accumulation of specific microRNAs in colorectal neoplasia. Mol Cancer Res 1: 882-891, 2003.

12. Lazebnik Y: What are the hallmarks of cancer? Nat Rev Cancer 10: 232-233, 2010.

13. Hanahan D and Weinberg RA: The hallmarks of cancer. Cell 100: 57-70, 2000.

14. Lopez-Camarillo C, Marchat LA, Arechaga-Ocampo E, et al: MetastamiRs: non-coding microRNAs driving cancer invasion and metastasis. Int J Mol Sci 13: 1347-1379, 2012.

15. Mendell JT and Olson EN: MicroRNAs in stress signaling and human disease. Cell 148: 1172-1187, 2012.

16. Schetter AJ, Leung SY, Sohn JJ, et al: MicroRNA expression profiles associated with prognosis and therapeutic outcome in colon adenocarcinoma. JAMA 299: 425-436, 2008. 
17. Pallante P, Visone R, Ferracin M, et al: MicroRNA deregulation in human thyroid papillary carcinomas. Endocr Relat Cancer 13: 497-508, 2006.

18. Zhai H and Ju J: Implications of microRNAs in colorectal cancer development, diagnosis, prognosis, and therapeutics. Front Genet 2: 00078, 2011.

19. Chen Y, Gelfond J, McManus LM, et al: Temporal microRNA expression during in vitro myogenic progenitor cell proliferation and differentiation: regulation of proliferation by miR- 682 . Physiol Genomics 43: 621-630, 2011.

20. Liu G, Min H, Yue S, et al: Pre-miRNA loop nucleotides control the distinct activities of mir-181a-1 and mir-181c in early T cell development. PLoS One 3: e3592, 2008.

21. Ji J, Yamashita T and Wang XW: Wnt/beta-catenin signaling activates microRNA-181 expression in hepatocellular carcinoma. Cell Biosci 1: 4, 2011.

22. Ren Y, Gao J, Liu JQ, et al: Differential signature of fecal microRNAs in patients with pancreatic cancer. Mol Med Rep 6 201-209, 2012.

23. Chen L, Yang Q, Kong WQ, et al: MicroRNA-181b targets cAMP responsive element binding protein 1 in gastric adenocarcinomas. IUBMB Life 64: 628-635, 2012.

24. Panarelli NC, Chen YT, Zhou XK, et al: MicroRNA expression aids the preoperative diagnosis of pancreatic ductal adenocarcinoma. Pancreas 41: 685-690, 2012.

25. Nurul-Syakima AM, Yoke-Kqueen C, Sabariah AR, et al: Differential microRNA expression and identification of putative miRNA targets and pathways in head and neck cancers. Int J Mol Med 28: 327-336, 2011

26. Ratert N, Meyer HA, Jung M, et al: Reference miRNAs for miRNAome analysis of urothelial carcinomas. PLoS One 7 : e39309, 2012.

27. Li X, Zhang $\mathrm{Y}$, Zhang $\mathrm{H}$, et al: miRNA-223 promotes gastric cancer invasion and metastasis by targeting tumor suppressor EPB41L3. Mol Cancer Res 9: 824-833, 2011.

28. Schaefer A, Jung M, Mollenkopf HJ, et al: Diagnostic and prognostic implications of microRNA profiling in prostate carcinoma. Int J Cancer 126: 1166-1176, 2010.

29. Calin GA and Croce CM: MicroRNA signatures in human cancers. Nat Rev Cancer 6: 857-866, 2006.

30. Gregory RI and Shiekhattar R: MicroRNA biogenesis and cancer. Cancer Res 65: 3509-3512, 2005.

31. Eis PS, Tam W, Sun L, et al: Accumulation of miR-155 and BIC RNA in human B cell lymphomas. Proc Natl Acad Sci USA 102: 3627-3632, 2005.

32. Cervigne NK, Reis PP, Machado J, et al: Identification of a microRNA signature associated with progression of leukoplakia to oral carcinoma. Hum Mol Genet 18: 4818-4829, 2009.

33. Frank DA: STAT3 as a central mediator of neoplastic cellular transformation. Cancer Lett 251: 199-210, 2007.

34. Yu H, Kortylewski M and Pardoll D: Crosstalk between cancer and immune cells: role of STAT3 in the tumour microenvironment. Nat Rev Immunol 7: 41-51, 2007.

35. Yu H, Pardoll D and Jove R: STATs in cancer inflammation and immunity: a leading role for STAT3. Nat Rev Cancer 9: 798-809, 2009.

36. Iliopoulos D, Jaeger SA, Hirsch HA, et al: STAT3 activation of miR-21 and miR-181b-1 via PTEN and CYLD are part of the epigenetic switch linking inflammation to cancer. Mol Cell 39: 493-506, 2010

37. Sun X, Icli B, Wara AK, et al: MicroRNA-181b regulates NF-kappaB-mediated vascular inflammation. J Clin Invest 122 1973-1990, 2012

38. Xi Y, Formentini A, Chien M, et al: Prognostic values of microRNAs in colorectal cancer. Biomark Insights 2: 113-121, 2006.

39. Nakajima G, Hayashi K, Xi Y, et al: Non-coding microRNAs hsa-let-7g and hsa-miR-181b are associated with chemoresponse to S-1 in colon cancer. Cancer Genomics Proteomics 3: 317-324, 2006.

40. Xu R, Ma N, Wang F, Ma L, et al: Results of a randomized and controlled clinical trial evaluating the efficacy and safety of combination therapy with Endostar and S-1 combined with oxaliplatin in advanced gastric cancer. Onco Targets Ther 6 : 925-929, 2013.
41. Watanabe K, Kawahara H, Enomoto H, et al: Feasibility Study of Oxaliplatin with Oral S-1 or Capecitabine as First-line Therapy for Patients with Metastases from Colorectal Cancer. Anticancer Res 33: 4029-4032, 2013

42. Jiang J, Zheng X, Xu X, et al: Prognostic significance of miR-181b and miR-21 in gastric cancer patients treated with S-1/oxaliplatin or doxifluridine/oxaliplatin. PLoS One 6: e23271, 2011.

43. Zhu W, Shan X, Wang T, et al: miR-181b modulates multidrug resistance by targeting BCL2 in human cancer cell lines. Int J Cancer 127: 2520-2529, 2010.

44. Wang B, Hsu SH, Majumder S, et al: TGFbeta-mediated upregulation of hepatic miR-181b promotes hepatocarcinogenesis by targeting TIMP3. Oncogene 29: 1787-1797, 2010.

45. Wang B, Li W, Guo K, et al: miR-181b promotes hepatic stellate cells proliferation by targeting p27 and is elevated in the serum of cirrhosis patients. Biochem Biophys Res Commun 421: 4-8, 2012.

46. Yan LX, Huang XF, Shao Q, et al: MicroRNA miR-21 overexpression in human breast cancer is associated with advanced clinical stage, lymph node metastasis and patient poor prognosis. RNA 14: 2348-2360, 2008

47. Mansueto G, Forzati F, Ferraro A, et al: Identification of a new pathway for tumor progression: microRNA-181b up-regulation and CBX7 down-regulation by HMGA1 protein. Genes Cancer 1: 210-224, 2010

48. Jones KB, Salah Z, Del Mare S, et al: miRNA signatures associate with pathogenesis and progression of osteosarcoma. Cancer Res 72: 1865-1877, 2012.

49. Xu X, Jia R, Zhou Y, et al: Microarray-based analysis: Identification of hypoxia-regulated microRNAs in retinoblastoma cells. Int J Oncol 38: 1385-1393, 2011.

50. Shi L, Cheng Z, Zhang J, et al: hsa-mir-181a and hsa-mir-181b function as tumor suppressors in human glioma cells. Brain Res 1236: 185-193, 2008.

51. Ciafre SA, Galardi S, Mangiola A, et al: Extensive modulation of a set of microRNAs in primary glioblastoma. Biochem Biophys Res Commun 334: 1351-1358, 2005.

52. Conti A, Aguennouz M, La Torre D, et al: miR-21 and 221 upregulation and miR-181b downregulation in human grade II-IV astrocytic tumors. J Neurooncol 93: 325-332, 2009.

53. Zhi F, Chen X, Wang S, et al: The use of hsa-miR-21, hsa-miR-181b and hsa-miR-106a as prognostic indicators of astrocytoma. Eur J Cancer 46: 1640-1649, 2010.

54. Slaby O, Lakomy R, Fadrus P, et al: MicroRNA-181 family predicts response to concomitant chemoradiotherapy with temozolomide in glioblastoma patients. Neoplasma 57: 264-269, 2010

55. Tunca B, Tezcan G, Cecener G, et al: Olea europaea leaf extract alters microRNA expression in human glioblastoma cells. J Cancer Res Clin Oncol 138: 1831-1844, 2012.

56. Calin GA, Dumitru CD, Shimizu M, et al: Frequent deletions and down-regulation of micro- RNA genes miR15 and miR16 at 13 q14 in chronic lymphocytic leukemia. Proc Natl Acad Sci USA 99: 15524-15529, 2002.

57. Chen H, Chen Q, Fang M, et al: MicroRNA-181b targets MLK2 in HL-60 cells. Sci China Life Sci 53: 101-106, 2010.

58. Zhu DX, Zhu W, Fang C, et al: miR-181a/b significantly enhances drug sensitivity in chronic lymphocytic leukemia cells via targeting multiple anti-apoptosis genes. Carcinogenesis 33: 1294-1301, 2012

59. Zanette DL, Rivadavia F, Molfetta GA, et al: miRNA expression profiles in chronic lymphocytic and acute lymphocytic leukemia. Braz J Med Biol Res 40: 1435-1440, 2007.

60. Sivina M, Hartmann E, Vasyutina E, et al: Stromal cells modulate TCL1 expression, interacting AP-1 components and TCL1-targeting micro-RNAs in chronic lymphocytic leukemia. Leukemia 26: 1812-1820, 2012. 